\title{
An analysis on the transmission of electron density through sulphur atoms in the quaternization reactions of benzothiazoles
}

\author{
B K MISHRA* and SABITA PATEL \\ Centre of Studies in Surface Science and Technology, Department of \\ Chemistry, Sambalpur University, Jyoti Vihar 768 019, India \\ e-mail: bijaym@hotmail.com
}

MS received 12 February 2001; revised 21 September 2001

\begin{abstract}
The kinetics of quaternization of a number of 2-substituted benzothiazoles with alkyl iodides and phenacyl bromide have been studied in nitrobenzene. The order of reactivity of different substituted benzothiazoles has been found to be $-\mathrm{H}>\mathrm{NO}_{2}>\mathrm{Cl}$. The relative reactivity of 2-amino and 2-methyl derivatives has been ascribed to a solvation phenomenon. The analysis of rho values from the Hammett equation suggests two possible routes for the transmission of electron density.
\end{abstract}

Keywords. Quaternization reaction; benzothiazole; Hammett equation.

\section{Introduction}

In continuation of our earlier studies on reactions on benzothiazole derivatives ${ }^{1-7}$ we report, herein, the quaternization kinetics of some benzothiazoles with alkyl iodides and phenacyl bromide in nitrobenzene medium. The pioneering work of Menschutkin ${ }^{8}$ on the quaternization reaction has initiated a good deal of work with varieties of nucleophiles. Deady $^{9}$, Grob et $a l^{10}$ and Behera et $a l^{11}$ have studied the quaternization kinetics of various heterocyclic compounds in different environments. While studying the quaternization kinetics of 2-aminobenzothiazoles with phenacyl bromide, Mishra et al have proposed the electron flow from a substituent to the reaction site through sulphur atom $^{2}$. In the present study, an attempt has been made to propose a quantitative approach to the electron flow from the substituent to the reacting nitrogen.

\section{Experimental}

\subsection{Preparation and purification of materials}

The preparation of 2-amino benzothiazole and its substituted ones is reported earlier ${ }^{11}$. 2-Methyl 6-substituted benzothiazoles were prepared by the oxidative cyclisation of appropriately substituted thioacetanilide in presence of hexacyanoferrate ${ }^{12}$ (2-methyl 6nitrobenzothiazole , MP $80^{\circ} \mathrm{C}$, yield $70 \%$, Found: N, $14.28 \% ; \mathrm{S}, 16 \cdot 38 \%$, calculated for $\mathrm{C}_{8} \mathrm{H}_{6} \mathrm{~N}_{2} \mathrm{O}_{2} \mathrm{~S}, \mathrm{~N}, 14.4 \% ; \mathrm{S}, 16.5 \%$, and 2-methyl 6-chlorobenzothiazole, MP $128^{\circ}$, yield $60 \%$, found: N, 7.54\%; S, 17.43\%; calculated for $\mathrm{C}_{8} \mathrm{H}_{6} \mathrm{NSCl}, \mathrm{N}, 7 \cdot 69 \%$; S, 17.6\%).

\footnotetext{
*For correspondence
} 
Phenacyl bromide was synthesised by bromination of acetophenones by the method already reported ${ }^{13}$. All the above compounds prepared were crystallized from alcohol and there homogeneity was checked by tlc on a silica gel plate.

Alkyl iodides were purified by the standard method ${ }^{14}$. 2-Methyl benzothiazole was obtained from Schudradt and Co. and was distilled under reduced pressure. Nitrobenzene $(\mathrm{AR} B \mathrm{BD})$ was purified and stored in dark ${ }^{15}$.

\subsection{Kinetic measurement}

The method of measuring rate of the reaction has been reported earlier ${ }^{3}$. Pseudo firstorder kinetics were maintained during the course of the kinetic run where the concentration of alkyl halide was around 20 times that of the substrate. The rate data at various temperatures and the values of $\Delta H^{\neq}$and $\Delta S^{\neq}$are given in tables 1 and 2.

\section{Results and discussion}

The reaction proceeds in the following manner.

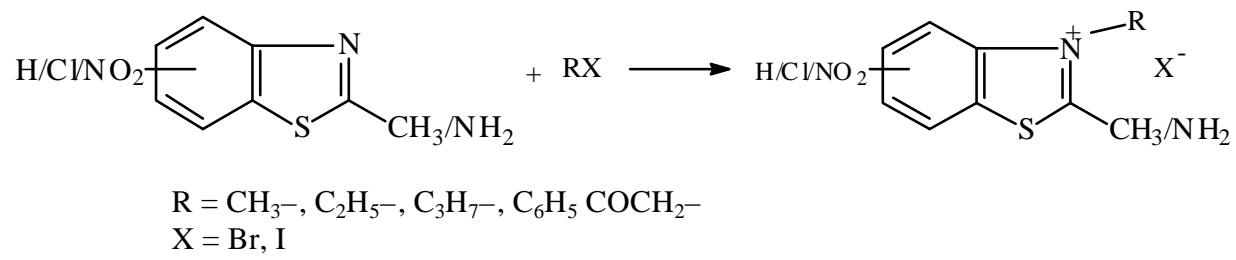

The reaction occurs at the ring nitrogen in preference to the exocyclic nitrogen, because of the existence of the charge delocalization over both nitrogens in the manner of an amidinium cation. The transition state for quaternization at the ring nitrogen is more stable than that at the exocyclic nitrogen. Therefore, the kinetic controlled product due to the quaternization at the most basic nitrogen is isolated. It is also known that mono alkylation and protonation of 2-aminopyridine $(p K a=7 \cdot 2)$ mainly occurs at the ring nitrogen ${ }^{16}$.

The formation of the ions due to quaternization has been followed conductometrically and from the slopes of the linear plots of $\log \left(R_{t} / R_{\infty}-R_{t}\right)$ vs time, the values of rate constant have been evaluated and given in tables 1 and 2 . With a view to checking the possibility of ion pair formation in nitrobenzene, which would cause an increase in $R_{\infty}$ value and give erroneous results of rate constants, the values of resistance of very dilute solutions of methiodides were obtained. The linear plot of resistance versus concentration on extrapolation gave the same value of $R_{\infty}$ at the desired reaction concentration.

Variously substituted 2-amino and 2-methyl benzothiazoles were quaternized with methyl iodide and phenacyl bromide. The order of reactivity of the substituents was found to be $-\mathrm{H}>\mathrm{NO}_{2}>\mathrm{Cl}$. The substituents at position 2, affect the reaction rate in the order, 2-Me $>2-\mathrm{NH}_{2}$ for all the three sets of compounds. This is quite in agreement with earlier observation ${ }^{11}$. The rate ratio of 2 -methyl vs 2 -amino compounds is found to be temperature independent. The values of rate ratio are 1.3 for benzothiazole, 2.6 for 6-chlorobenzothiozole and 2.0 for 6-nitrobenzothiozole systems. The values of enthalpy of activation varies between 37 to $64 \mathrm{~kJ}$ per mole and those of entropy of activation between -150 joule to -250 joule. 


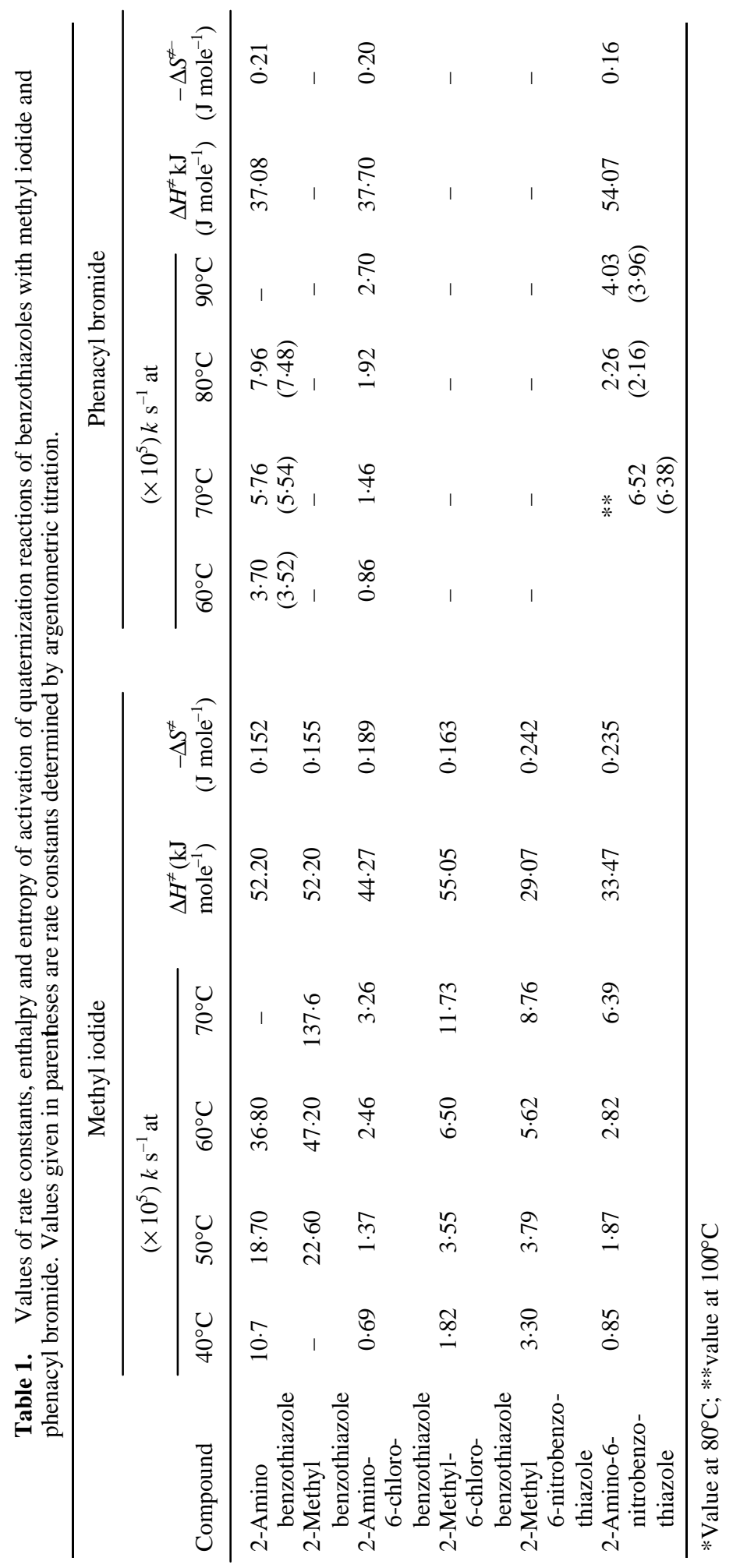


Table 2. Values of rate constants, enthalpy and entropy of activation of the quaternization reaction of benzothiazoles with alkyl iodides.

\begin{tabular}{|c|c|c|c|c|c|c|c|}
\hline \multirow[b]{2}{*}{ Benzothiazoles } & \multirow{2}{*}{$\begin{array}{l}\text { Alkyl } \\
\text { iodides }\end{array}$} & \multicolumn{4}{|c|}{$\left(\times 10^{5}\right) k$ in s ${ }^{-1}$} & \multirow{2}{*}{$\begin{array}{c}\Delta H^{\neq} \\
\left(\mathrm{kJ} \mathrm{mole}^{-1}\right)\end{array}$} & \multirow{2}{*}{$\begin{array}{c}-\Delta S^{\#} \\
\left(\mathrm{~kJ} \mathrm{~mole}^{-1}\right)\end{array}$} \\
\hline & & $40^{\circ} \mathrm{C}$ & $50^{\circ} \mathrm{C}$ & $60^{\circ} \mathrm{C}$ & $70^{\circ} \mathrm{C}$ & & \\
\hline $\begin{array}{l}\text { 2-Amino } \\
\text { benzothiazole }\end{array}$ & Ethyl iodide & 0.037 & $0 \cdot 051$ & $0 \cdot 176$ & $0 \cdot 199$ & $50 \cdot 28$ & $0 \cdot 189$ \\
\hline $\begin{array}{l}\text { 2-Methyl } \\
\text { benzothiazole }\end{array}$ & Ethyl iodide & $0 \cdot 041$ & $0 \cdot 053$ & $0 \cdot 085$ & $0 \cdot 162$ & $63 \cdot 81$ & $0 \cdot 158$ \\
\hline $\begin{array}{l}\text { 2-Amino } \\
\text { benzothiazole }\end{array}$ & Propyl iodide & $0 \cdot 015$ & $0 \cdot 087$ & 0.097 & $0 \cdot 142$ & $62 \cdot 85$ & $0 \cdot 156$ \\
\hline $\begin{array}{l}\text { 2-Methyl } \\
\text { benzothiazole }\end{array}$ & Propyl iodide & $0 \cdot 192^{*}$ & $0 \cdot 046$ & $0 \cdot 066$ & $0 \cdot 121$ & $45 \cdot 45$ & $0 \cdot 211$ \\
\hline
\end{tabular}

The reactivity of nitrogen atom is influenced by the substituent possibly by two paths one through sulphur atom and the other directly. Both the paths of propagation of electron density for substituents at position 5 and 6 are shown in I for 5- and 6-substituted benzothiazoles. For a similar situation encountered in the alkaline hydrolysis of phthalides, Jaffe ${ }^{17}$ has used two reaction constants for the difference in the electron propagation path from the substituent to the reaction site. He has proposed an equation

$$
\log k / k_{\mathrm{o}}=\rho_{1} \sigma_{m}+\rho_{2} \sigma_{P}
$$

where $\rho_{1}$ and $\rho_{2}$ are two different reaction constants for the two different paths of electron propagation from the substituent placed at a position meta and para to the propagation path respectively.

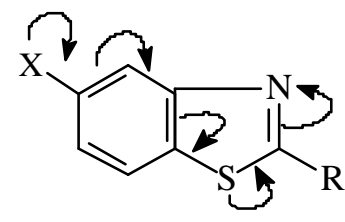

(a)

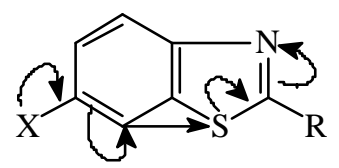

(c)<smiles></smiles>

(b)

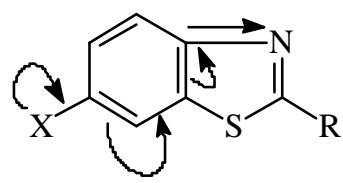

(d)

(I)

In analogy to Hammett-Jaffe equation, two equations (2) and (3) can be written where $\rho_{s}$ and $\rho_{d}$ are the reaction constants for transmission of electron through sulphur or through a direct path and $k_{5}$ and $k_{6}$ are reaction constant for 5 and 6 substituents 
Table 3. Values of $\rho_{s}$ and $\rho_{d}$ for the reaction of (i) 2-amino benzothiazoles + methyl iodide, (ii) 2-methyl benzothiazoles + methyl iodide, and (iii) 2-amino benzothiazoles + phenacyl bromide.

\begin{tabular}{lcrc}
\hline Reaction & $\rho_{s}$ & $\rho_{d}$ & $\left(\rho_{s}+\rho_{d}\right)$ \\
\hline (i) & -2.58 & 0.92 & -1.66 \\
(ii) & -6.11 & 4.37 & -1.74 \\
(iii) & -0.94 & -0.15 & -1.09 \\
\hline
\end{tabular}

respectively. In I-a (sulphur route) and I-d (direct route) the substituents behave as para substituents in the benzene ring where as in $\mathrm{I}-\mathrm{b}$ and $\mathrm{I}-\mathrm{c}$ they behave as meta substituents.

$$
\begin{aligned}
& \log k_{5} / k_{H}=\rho_{d} \sigma_{m}+\rho_{s} \sigma_{P}, \\
& \log k_{6} / k_{H}=\rho_{d} \sigma_{P}+\rho_{s} \sigma_{m} .
\end{aligned}
$$

The values of $\rho_{\mathrm{s}}$ and $\rho_{\mathrm{d}}$ have been obtained for the reaction of (i) 2-aminobenzothiazoles and methyliodide, (ii) 2-methylbenzothiazoles and methyliodide, and (iii) 2aminobenzothiazoles and phenacyl bromide (table 3 ). The rho values have been evaluated with a view to see the trend in the changes of rho values in the three sets of reactions. The trends in these values lead to some striking conclusions.

1. There is a greater flow of electron density to nitrogen through sulphur route than through a direct route. Davis et $a l^{18}$ have also indicated substantial transmission of electronic effects through the sulphur atom from the ultraviolet spectral data of some sulphur amides. A mechanism of through-conjugation involving $p \pi-\mathrm{d} \pi-p \pi$ conjugation have been suggested for phenyl vinyl sulphides and supported by CNDO/2 calculations ${ }^{19}$.

2. The demand of the transition state on the substituent in benzothiazole is more in 2-methyl compounds than in 2-amino compounds. This is quite obvious since an amino group stabilizes the developing positive charge much more than a methyl group does $\left(\sigma_{\mathrm{P}-\mathrm{Me}}=-0.31\right.$ and $\left.\sigma_{\mathrm{P}-\mathrm{NH}_{2}}=-1 \cdot 3\right)$.

3. Further in 2-amino compounds the demand of the transition state on the substituents is very much less in the reaction with phenacyl bromide than with methyl iodide. This may be due to the formation of a hydrogen bonded cyclic transition state with phenacyl bromide ${ }^{3}$.

4. The kinetics of quaternization of 2-methyl benzothiazole has also been followed with ethyl and $n$-propyl iodides in nitrobenzene. The values of rate constants, enthalpy of activation and entropy of activation are given in table 2 . The order of reactivity of these alkyl iodides is MeI $>$ EtI $>n$-PrI. This order has also been observed earlier by Deady and Stillman ${ }^{20}$.

\section{Acknowledgement}

One of the authors (BKM) thanks the University Grants Commission, New Delhi for financial assistance through UGC Research Award for the 9th plan period. 


\section{References}

1. Mishra P, Mishra B K and Behera G B 1988 Indian J. Chem. A27 889

2. Mishra B K, Sharma A and Behera G B 1989 Int. J. Chem. Kinet. 21439

3. Mishra P K, Mishra B K and Behera G B 1991 Int. J. Chem. Kinet. 23639

4. Mishra P K, Mishra B K and Behera G B 1992 Int. J. Chem. Kinet. 24533

5. Senapati S, Dash P K, Mishra B K and Behera G B 1995 Indian J. Chem. A34 278

6. Mishra R K, Hota S, Mishra B K and Behera G B 1998 Indian J. Chem. A37 200

7. Patel N, Hota S, Mishra B K and Behera G B 2000 Indian J. Chem. A39 763

8. Menschutkin N 1890 Z. Phys. Chem. 5589

9. Deady L W 1973 Aust. J. Chem. 191949

10. Grob C A and Schlageter 1974 Helv. Chem. Acta 57509

11. Behera G B, Kar J N, Acharya R C and Rout M K 1973 J. Org. Chem. 38 2164; Behera G B and Sharma A 1979 Bull. Chem. Soc. Jpn 52604

12. Bhargava P N and Baliga B J 1958 J. Indian Chem. Soc. 35807

13. Konig W 1968 Ber. Dtsch. Chem. Ges. 612065

14. Browit E I and Prones H 1967 J. Org. Chem. 323560

15. Riddiel and Bunger (eds) 1970 Organic solvents: Physical properties and methods of purification (London: Wiley Interscience) vol 2, p 790

16. Joule J A and Smith G F 1978 In Heterocyclic chemistry (London: Van Nostrand Reinhold)

17. Jaffe H H 1953 Chem. Rev. 53191

18. Davis F A, Kaminishi J M, Kinger E W and Freilich H S 1975 J. Am. Chem. Soc. 977085

19. Kajimoto O, Koboyashi M and Feuno T 1973 Bull. Chem. Soc. Jpn 462316

20. Deady L W and Stillman D C 1976 Aust. J. Chem. 291745 